\title{
Probability of Incipient Spanning Clusters in Critical Two-Dimensional Percolation
}

\author{
L.N. Shchur* and S.S. Kosyakov \\ Landau Institute for Theoretical Physics, 142432 Chernogolovka, Russia
}

The probability of simultaneous occurence of at least $k$ spanning clusters has been studied by Monte Carlo simulations on the $2 \mathrm{D}$ square lattice at the bond percolation threshold $p_{c}=1 / 2$. The calculated probabilities for free boundary conditions and periodic boundary conditions are in a very good coincidence with the exact formulae developed recently by Cardy.

\section{INTRODUCTION}

It was a common belief until a very recent time that on two-dimensional (2D) lattices at percolation threshold $p_{c}$ there exists exactly one percolation cluster [1.2]. New insight developed recently by Aizenman, who proved [3] that the number of Incipient Spanning Clusters (ISC) in 2D critical percolation can be larger than one, and that the probability of at least $k$ separate clusters is bounded $A e^{-\alpha k^{2}} \leq P_{L}(k) \leq e^{-\alpha^{\prime} k^{2}}$, where $\alpha$ and $\alpha^{\prime}$ are constants and $L$ is a linear lattice size. We investigate by Monte-Carlo the number of spanning clusters in the critical bond percolation model on $2 \mathrm{D}$ square lattices. Using simple finite-size scaling of probabilities on a selfdual lattices of moderate size, we have determined with a good accuracy values of the probabilities $P(k)=\lim _{L \rightarrow \infty} P_{L}(k)$ for $k=1,2$ and 3.

\section{LATTICE}

We use in simulations a rectangular square lattice with linear size $L$, and exactly $L$ sites and $L$ bonds both horizontally and vertically. For clarity the example of lattice is shown in Fig. 11 together with the dual lattice. The dual lattice has the same number of sites and bonds as the original lattice. This gives the possibility to keep in the finite lattices some properties of infinite lattice. First of all, the number of bonds is exactly

\footnotetext{
*E-mail address: lev@itp.ac.ru. Partialy supported by grants RFBR 96-02-18168 and 96-07-89266, NWO 07-210 and INTAS 93-211
}

twice the number of sites. Second, the self-duality

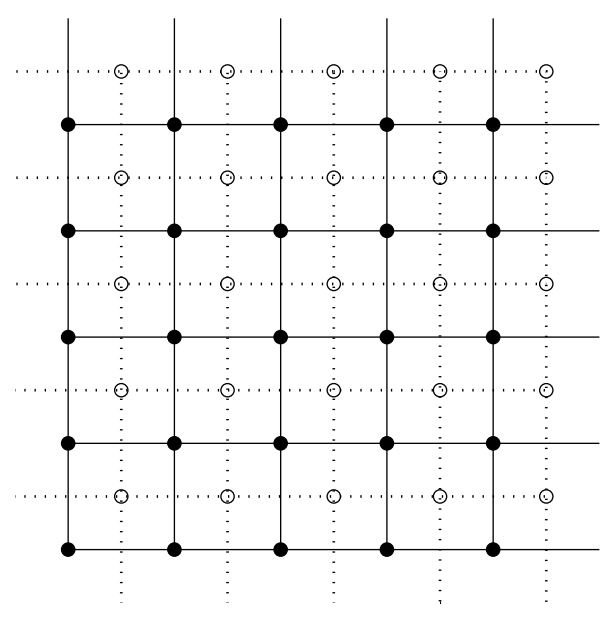

Figure 1. Example of lattice with $L=5$.

is valid for any finite lattice size. Third, the horizontal and vertical directions are equivalent. Finally, one could expect trivial finite-size scaling for the bond percolation on such lattices. Indeed, probabilities are proportional to the inverse system "volume", i.e. $\propto 1 / L^{2}$ (Fig. 2 and Fig. 3).

\section{ALGORITHM}

Each bond could be occupied with probability $q=1-p$ and closed with the probability $p$. 
Given the realization to each of $2 \cdot L^{2}$ bonds to be occupied formed the sample. Each sample could be exactly decomposed in clusters of connected occupied bonds. We are interested in the spanning properties of such a clusters. Namely, what is the probability that a cluster connects the opposite borders of square and what is the number of disjoint spanning clusters? It should be noted

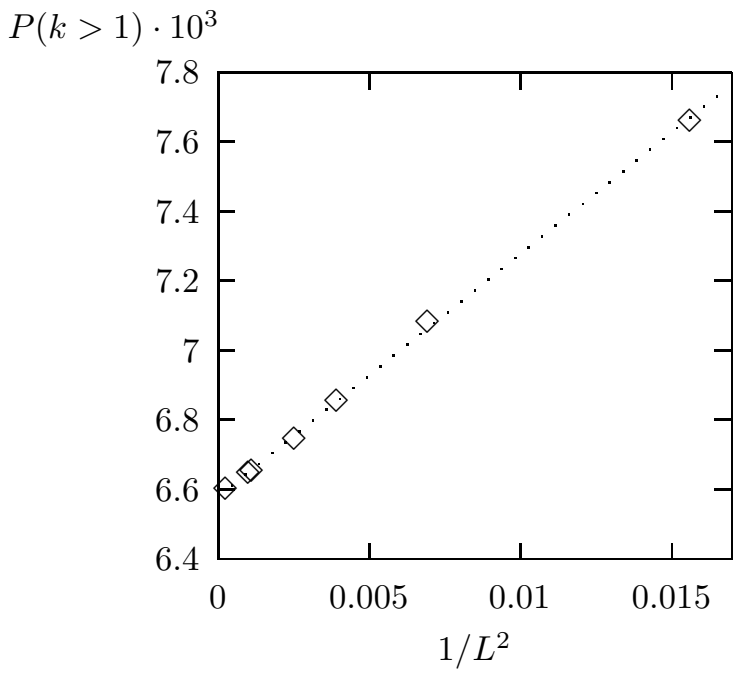

Figure 2. Probability of more than one Incipient Spanning Cluster.

that the variance of probabilities is independent from the lattice size because probabilities are calculated as the expectation values of corresponding indicator functions. So, we should keep the number of samples independent of the lattice size and, therefore, the only parameter which controls the statistical errors is the number of samples $M$. Probabilities were calculated averaging $M=10^{8}$ samples and the error bars were defined from 100 bins, each bin being the average over $10^{6}$ samples.

\section{NUMERICAL RESULTS}

We calculate the crossing probabilities $\pi_{h_{k}}$ and $\pi_{v_{k}}$ for square lattices with linear sizes $L=$
$8,12,16,20,30,32,64$ looking for all events up to $k=8$. See for details the more full text 任. On the Fig. 2 we plot the probability for simultaneous occurence of more than one spanning cluster $P_{L}(k>1)=\sum_{k>1} \pi_{h_{k}}$ versus the inverse system volume $1 / L^{2}$. A linear fit gives us the limiting value of $P(k>1)=6.58 \cdot 10^{-3}$ with error $\approx 3 \cdot 10^{-5}$. Fig. 3 shows the dependence

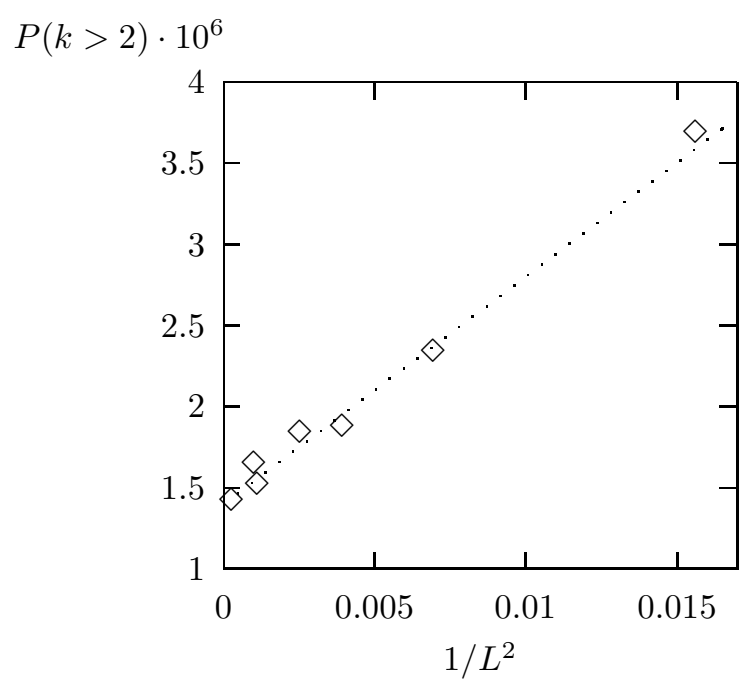

Figure 3. Probability of more than two Incipient Spanning Cluster.

of the probability of more than two clusters versus $1 / L^{2}$. The best linear fit gives the limiting value of $P(k>2)=1.48 \cdot 10^{-6}$ with uncertainty $2.1 \cdot 10^{-7}$.

Actually, we observed in computations mostly the events of up to three simultaneous spanning clusters. We simulated at total about $10^{10}$ samples of different sizes $8 \leq L \leq 64$ and only one sample with 4 spanning clusters was detected. This event clearly not contradicts with our estimate [4 for $P(4) \approx 10^{-11}$. Single event is probable as one part in ten.

A best linear fit to the logarithm of $P(k)$ versus $k^{2}$ gives $\alpha \propto 1.61(7)$ and $A \propto 3 \pm 1.5$. 
The crossing probability with periodic boundary conditions (PBC) is known to be larger and the value $0.63665(8)$ is computed in [6]. We estimate in computer simulations the probability of disjoint ISC clusters to be $P(k>1) \approx 2.0(4) 10^{-3}$ and $P(k>2) \approx 1.4(5) 10^{-7}$ which are even smaller than for the case of free boundary conditions (FBC) considered by us. The finite size scaling of PBC is more complicated [6] and fit of the results for only four lattice sizes $(8,16,32,64)$ we simulated gives less accurate limiting values in comparison with the one for FBC. Surprisingly for us, the linear fit of logarithm of $P(k)$ versus $k^{2}$ gives better accuracy then for the case of FBC: $\alpha_{P B C} \approx 1.915(1)$ and $A \approx 4.26(3)$. The different behaviour of probabilities will be explained in the next section.

\section{COMPARISON WITH EXACT RE- SULT}

Quite recently, John Cardy, using methods of conformal field theory, conjectured an exact form 5] of probability of $k$ distinct Incipient Spanning Clusters in the limit of large aspect ratio $r$ for rectangle (in our notations, $\mathrm{FBC}$ - free boundary conditions) and cylinder (PBC - periodic boundary conditions). It is possible to compare the data of our paper with the ratio, developed from formulae (2) and (3) of Cardy preprint

$$
\frac{\log P_{F B C}(k)}{\log P_{P B C}(k)}=\frac{k\left(k-\frac{1}{2}\right)}{k^{2}-\frac{1}{4}},
$$

which is $\frac{4}{5}=0.8$ for $k=2$ and $\frac{6}{7} \approx 0.857$ for $k=3$. Our data gives $0.808(10)$ and $0.851(20)$, correspondingly. The discussion of that close agreement can be found in Cardy's preprint. The difference in the $k$-dependence explains why our $\mathrm{PBC}$ data fits more closely to $k^{2}$ than the FBC ones. Therefore, using the known $k$-dependence of the probabilities, we can collapse all but $k=1$ data on a single curve (Fig.(4) versus a Cardy variable $X$ which is $X_{P B C}=k^{2}-1 / 4$ for periodic boundaries and $X_{F} B C=k^{2}-k / 2$ for free boundaries. The linear fit should give us an approximation of the exponent $\alpha_{\text {asymp }}$ in (2), which is $2 \pi / 3=2.094$ by Cardy's conjecture. The linear fit to our data gives 1.91(20), which is, again, not

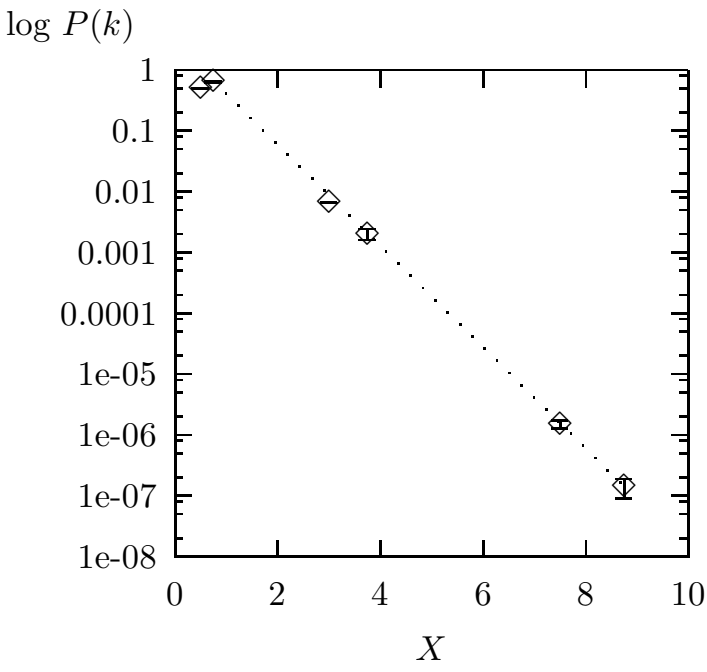

Figure 4. Probabilities of $k=1,2$ and 3 simultaneous clusters on lattices with periodic and free boundary conditions. $X_{P B C}=k^{2}-1 / 4$ and $X_{F B C}=k^{2}-k / 2$, correspondingly.

so bad an approximation to Cardy's asymptotic value.

It seems the fact that there are nonzero probability (about half of percent) of the coexistence of infinite clusters of black (open) and white (closed) sites (bonds) in critical two-dimensional percolation.

\section{REFERENCES}

1. D. Stauffer and A. Aharony, Introduction to Percolation Theory, (Taylor \& Francis, London, 1992).

2. G. Grimmet, Percolation, (Springer Verlag, New York, 1989)

3. M. Aizenman, Nucl. Phys. B [FS] 485 (1997) 551

4. L.N. Shchur and S.S. Kosyakov, Int.J. Mod.Phys. C8 (1997) 473

5. J. Cardy, preprint cond-mat/9705137

6. J.-P. Hovi and A. Aharony, Phys. Rev. E, 53 (1996) 235 